\title{
Síntesis, caracterización y evaluación catalítica de MOF UiO-66 en la esterificación de ácido levulínico
}

\section{Synthesis, characterization and catalytic evaluation of MOF UiO- 66 in the esterification of levulinic acid}

Presentación: 11/10/2019

\section{Doctorando:}

\section{Daiana Antonella, Bravo Fuchineco}

Centro de Investigación y Tecnología Química (CITeQ) / Consejo Nacional de Investigaciones Científicas y Técnicas (CONICET) / Universidad Tecnológica Nacional- Facultad Regional Córdoba (UTN-FRC), Maestro M. López esq. Cruz Roja Argentina - Edificio de Laboratorios $1^{\circ}$ Piso, 5000, Córdoba, Argentina.

dbravo@frc.utn.edu.ar

\section{Director/es:}

\section{Directora: Mónica Elsie, Crivello}

Co-directora Tesis: Angélica Constanza, Heredia

Co-director Beca: Enrique, Rodríguez Castellón

\section{Resumen}

El estudio de los compuestos metal-orgánicos MOFs ha experimentado un rápido crecimiento en las últimas dos décadas debido a sus interesantes propiedades: flexibilidad en el diseño y funcionalización de sus poros, elevada superficie específica y síntesis menos complejas. Dentro de esta gran familia de materiales, los que contienen $\mathrm{Zr}$ como metal activo, rodeado por ligandos de ácido tereftalico (UiO-66) son buenos catalizadores y estables para la esterificación de ácido levulínico con etanol, con actividades comparables a otros catalizadores ácidos sólidos. El objetivo del presente trabajo fue sintetizar compuestos del tipo MOF UIO-66 con circonio como precursor metálico y tetracloruro de circonio como fuente metálica, empleando una ruta alternativa de síntesis, con condiciones más favorables desde el punto de vista económico y ambiental. Es por ello que se propone el uso de solventes alternativos. La síntesis se realizó mediante el método solvotermal a $120{ }^{\circ} \mathrm{C}$ durante $24 \mathrm{hs}$. Se utilizó dimetilformamida como solvente y se reemplazó entre un 0 y 75\%, de dicho solvente, por acetona. Estos materiales luego se evaluaron catalíticamente en la reacción de esterificación del ácido levulínico con etanol. Las propiedades del material fueron caracterizadas por difracción de rayos X, microscopía electrónica de barrido, espectroscopia infrarroja, espectroscopía fotoelectrónica de rayos X, espectroscopia de emisión atómica por plasma de microondas y área superficial. El progreso de la reacción fue seguido por cromatografía gaseosa y espectroscopía de masa. El material más activo fue el $\mathrm{MOF}_{100 \%}$ con 17,42\% de conversión y 27,17 \% de selectividad a levulinato de etilo.

Palabras claves: Red metal-orgánica (MOF), UIO-66, solvotermal, biomasa, ácido levulínico, esterificación.

\begin{abstract}
The study of the metal-organic frameworks MOFs has experienced rapid growth over the last two decades due to their interesting properties: flexibility of design and functionalization of their pores, high specific surface area and less complex synthesis. Within this large family of materials, those containing $\mathrm{Zr}$ as active metal, surrounded by terephthalic acid ligands (UiO-66) are good catalysts and stable for the esterification of levulinic acid with ethanol, activities comparable to other catalysts directly. The objective of this work was synthesized compounds of the MOF UIO-66 type with zirconium as a metal precursor and zirconium tetrachloride as a metallic source, using an alternative route of synthesis, with more favorable conditions from the economic and environmental point of view. That is why the use of alternative solvents is proposed. The synthesis was carried out using the solvotermal method at $120^{\circ} \mathrm{C}$ for 24 hours. Dimethylformamide was considered as solvent and between 0 and $75 \%$ of said solvent was replaced by acetone. These materials were then catalytically evaluated in the esterification reaction of levulinic acid with ethanol. The properties of the material were characterized by X-ray diffraction, scanning electron microscopy, infrared spectroscopy, X-ray photoelectronic spectroscopy, microwave plasma atomic emission spectroscopy and superfical area. The progress of the reaction was followed by gas chromatography and mass spectroscopy. The most active material was MOF100\% with $17,42 \%$ conversion and $27,17 \%$ selectivity to ethyl levulinate.
\end{abstract}

Keywords: Metal-organic network (MOF), UIO-66, solvotermal, biomass, levulinic acid, esterification. 


\section{Introducción}

La transformación catalítica y selectiva de compuestos derivados de la biomasa, es una tecnología clave en la producción de forma sostenible. El ácido levulínico ha sido identificado como una plataforma química prometedora [1]. Se puede obtener a partir de carbohidratos y biomasa a base de celulosa. Los ésteres de levulinato poseen la baja toxicidad, alta lubricidad, estabilidad del punto de inflamación y propiedades de flujo moderado en condiciones de baja temperatura, lo que hace que sean aditivos adecuados para combustibles de gasolina y diesel [2].

El etil levulinato generalmente se sintetiza utilizando catalizadores ácidos homogéneos como el $\mathrm{H}_{2} \mathrm{SO}_{4}$, el $\mathrm{HCl}$ y el $\mathrm{H}_{3} \mathrm{PO}_{4}$ generando inconvenientes en el reciclaje del catalizador, la separación de productos y problemas ambientales. Los catalizadores ácidos sólido heterogéneos son una buena alternativa para superar los inconvenientes de los homogéneos, pueden separarse de las mezclas de reacción fácilmente y reutilizarse para ensayos repetidos. [3].

Los materiales metal orgánico estructurados (MOFs) pertenecen a la familia de los híbridos cristalinos formados por una red de iones metálicos enlazados a moléculas orgánicas multidentadas mediante enlaces de coordinación. La combinación de unidades de construcción orgánicas e inorgánicas da lugar a estructuras cristalinas, en muchos casos porosas, y con una elevada variabilidad en cuanto a composición química, tamaño de poro y grupos funcionales presentes en el ligando orgánico [4]. Poseen características como elevada superficie específica, buena estabilidad térmica y química, lo que otorga un gran potencial de aplicación en adsorción, separación y catálisis. Es importante remarcar que las propiedades catalíticas de estos materiales están directamente relacionadas con el metal en la estructura [5].

El MOF UiO-66 fue sintetizado por primera vez en 2008 por científicos de la Universitetet i Oslo (UiO) utilizando un tratamiento solvotérmico a $120{ }^{\circ} \mathrm{C}$ en dimetilformamida como solvente [6]. Aquí el circonio unido a la red orgánica de ácido tereftalico, confiere propiedades específicas para distintos tipos de reacciones orgánicas. Además, estos MOFs presentan buena estabilidad térmica y mecánica; son estables en agua y en disolventes orgánicos comunes, debido a la presencia del grupo $\mathrm{Zr}_{6} \mathrm{O}_{4}(\mathrm{OH})_{4}$ como bloques de construcción inorgánicos. Las caras triangulares del octaedro $\mathrm{Zr}_{6}$ en esta estructura están alternativamente cubiertas con oxígenos e hidroxilos que les confieren características de acidez de Lewis y Brønsted, requeridas para las reacciones de esterificación. [7]

La esterificación del ácido levulínico con el etanol catalizada heterogéneamente es un proceso verde muy atractivo. Es por ello que la presente investigación se ha centrado en la síntesis de redes metal-orgánicas del tipo UiO-66 mediante el método solvotermal, variando la concentración del solvente. Se buscó la manera de mejorar el método de síntesis para obtener un material con un alto rendimiento y buenas propiedades porosas en condiciones más eco-compatibles.

\section{Resultados}

\section{$>$ Materiales y Métodos}

\section{Reactivos}

Para la preparación de los catalizadores y las reacciones catalíticas se utilizaron reactivos de grado analítico. $\mathrm{ZrCl}_{4}$ (Merck, $\geq 98 \%$ ), ácido tereftálico (BDC, Aldrich, 98\%), N,N-dimetilformamida (DMF, Biopack, $\geq 99,8 \%$ ), Acetona (Sintorgan, 99,5\%), Etanol (Biopack), Ácido Levulínico (Aldrich, 98\%).

\section{Síntesis del material}

Los materiales fueron sintetizados mediante el método solvotermal siguiendo lo descrito por Cavka y col. (2008) [8]. Se realizaron cuatro síntesis variando el porcentaje del solvente (DMF) desde 25 a $100 \%$ con acetona. Las mismas fueron identificadas como MOF $\mathrm{x} \%$, siendo $\mathrm{X}$ el contenido de DMF.

El procedimiento para la síntesis consistió en disolver $1,083 \mathrm{~g} \mathrm{de} \mathrm{ZrCl}_{4}$ en $130 \mathrm{~mL}$ del solvente (DMF y/o acetona); agitando magnéticamente la solución por 5 minutos. Luego se incorporaron 1,069 g de ácido tereftálico (BDC). La mezcla completa se mantuvo en agitación durante 30 minutos. A continuación, se colocó la solución en un autoclave en una estufa precalentada a $120{ }^{\circ} \mathrm{C}$ durante $24 \mathrm{~h}$. Pasado dicho tiempo, se lo dejó enfriar hasta temperatura ambiente. El material obtenido se sumergió en DMF y luego se lavó con acetona, con el objetivo de intercambiar la DMF por un disolvente con un menor punto de ebullición, que pueda ser eliminado fácilmente. El sólido formado se separó del disolvente por filtración, obteniéndose un sólido pulverulento de color blanco. Por último, el material se secó a $90^{\circ} \mathrm{C}$ por $24 \mathrm{~h}$.

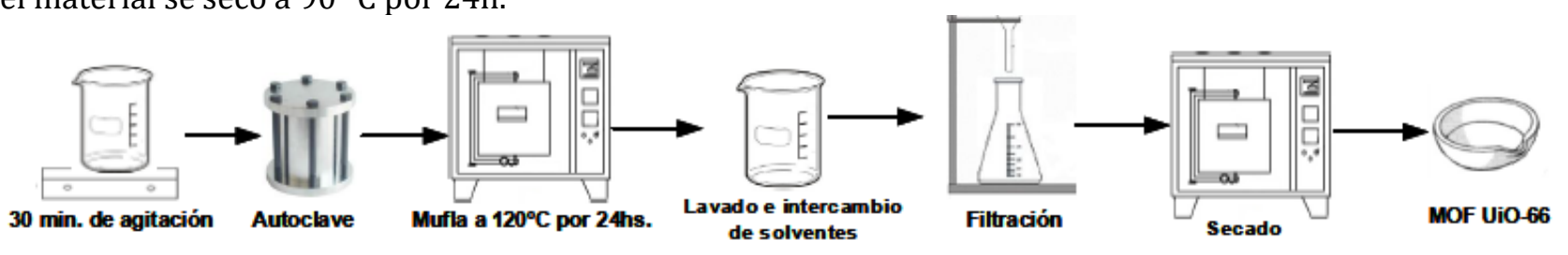

Figura 1- Sistema de síntesis del catalizador 


\section{Caracterización de las muestras}

Difracción de rayos X (DRX): los materiales estudiados en este trabajo fueron caracterizados cristalográficamente mediante rayos X (DRX), haciendo uso de un difractómetro Xpert' PANanalytical de radiación $\mathrm{Cu}-\mathrm{K} \alpha$, a una velocidad de 1/4 min en 20. Espectroscopia infrarroja (FTIR): los espectros de las muestras compactadas con $\mathrm{KBr}$, se registraron en el rango de 4000-400 $\mathrm{cm}^{-1}$ usando un espectrofotómetro Smartomi-Transmission Nicolet IS10 Thermo Scientific. Microscopía electrónica de barrido (SEM): los análisis de SEM fueron realizados mediante un instrumento Carl Zeiss Sigma operado a $2 \mathrm{Kv}$. Las muestras fueron metalizadas con oro. Medición del área superficial: el área superficial fue determinada utilizando un instrumento ASAP 2000 (Micromeritics, EE. UU.) y calculada mediante el método Brunauer-Emmett-Teller (BET). Antes de la determinación de las isotermas de adsorción, los precursores se trataron a $200^{\circ} \mathrm{C}$ y las muestras calcinadas a $390^{\circ} \mathrm{C}$, durante 60 minutos bajo vacío de $1.0 \times 10^{-3}$ mbar. Espectroscopía fotoelectrónica de rayos X (XPS): el estado químico y la estructura electrónica de los elementos de los materiales fue determinado con XPS Thermo Scientific K-alpha. Espectroscopia de emisión atómica por plasma de microondas (MP-AES): se realizó el análisis elemental con un MP-AES, marca Agilent, modelo 4200.

\section{Reacción Catalítica}

Con el fin de evaluar los materiales metal-orgánicos de $\mathrm{Zr}$ como catalizadores ácidos para la reacción de esterificación, se colocó ácido levulínico y etanol en una relación 1:15 junto con 0,05 gr del catalizador en un reactor batch de vidrio acoplado a un condensador de reflujo. Dicho sistema se sumergió en un baño con agitación para mantener la temperatura en $78{ }^{\circ} \mathrm{C}$ [9]. La reacción se desarrolló en $5 \mathrm{~h}$ y se tomaron muestras cada hora. El progreso se controló por GC (sistema GC 7820A de Agilent Technologies equipado con HP-20M $25 \mathrm{~m}, 0,20 \mathrm{~mm}$, columna de $0,10 \mu \mathrm{m}$ y detector FID). Posteriormente se realizó espectroscopía de masa en un equipo Perkin Elmer Clarus 560 GC-MS, para confirmar la identificación de los compuestos.

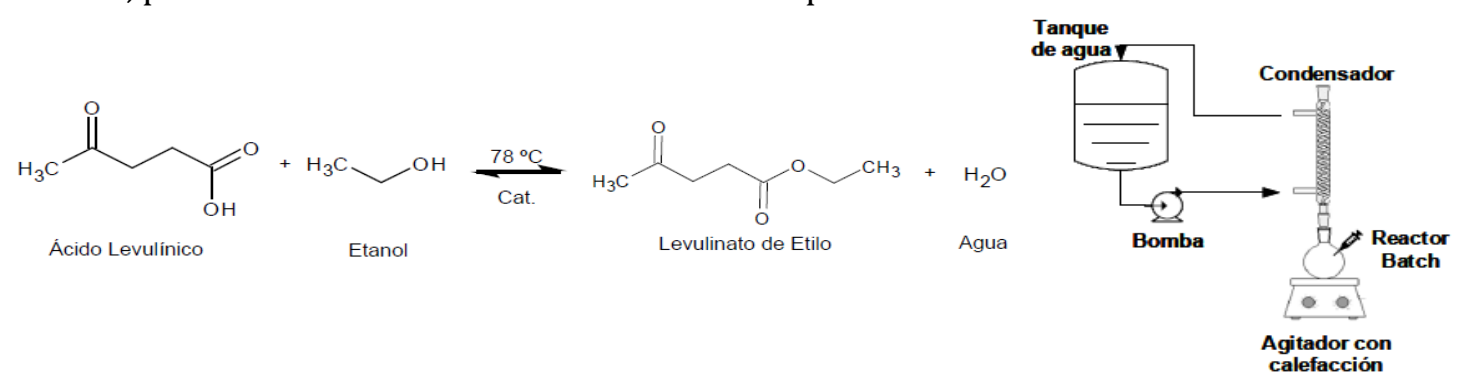

Figura 2- Reacción de esterificación y sistema de reacción

\section{$>$ Caracterización físico-química}

La figura 3 muestra el patrón de difracción de rayos X de los materiales sintetizados, con la variación del contenido del solvente (DMF) de 25 a 100\%. Se pueden observar 2 señales producidas a los $7,4^{\circ}$ y $8,5^{\circ}$ las cuales son asignadas a los planos cristalográficos $\left(\begin{array}{llll}1 & 1 & 1\end{array}\right)$ y $\left(\begin{array}{lll}2 & 0 & 0\end{array}\right)$ de la estructura MOF UIO-66. Por DRX, se determinó que al reducir la cantidad de DMF los materiales presentaron una menor cristalinidad, tal como se observa en la variación de la línea de base.

La estructura química de los materiales se analizó por FTIR. En la figura 4 se muestran las señales para el grupo COO- (1585 y $\left.1397 \mathrm{~cm}^{-1}\right)$ asociados con las vibraciones de estiramiento asimétrica y simétrica en el grupo carboxilo de la BDC y una banda más pequeña $\left(1506 \mathrm{~cm}^{-1}\right)$ que representa la vibración del $\mathrm{C}=\mathrm{C}$ del anillo bencénico. Las dos señales de menor frecuencia (a los 548 y $483 \mathrm{~cm}^{-1}$ ) pueden deberse a los estiramientos $\mathrm{Zr}-\mathrm{O}$ en el cluster del MOF.

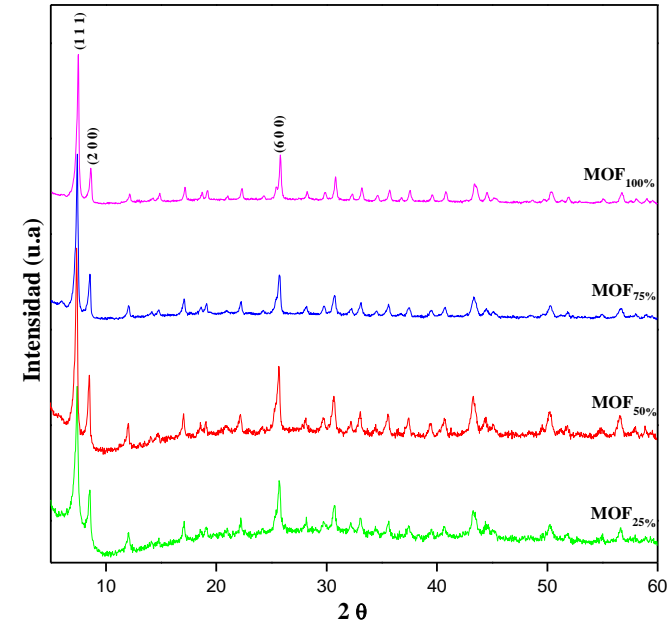

Figura 3 - Difractogramas de RX de los sólidos sintetizados.

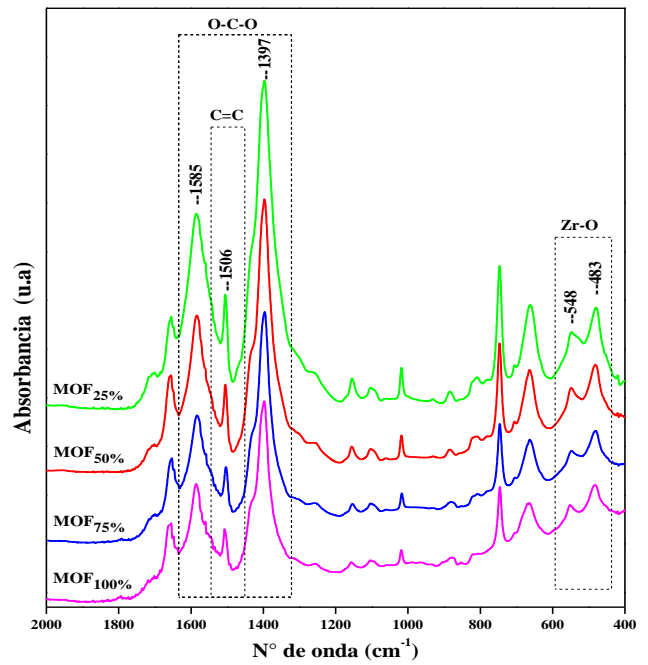

Figura 4 - Espectros FTIR de los sólidos sintetizados. 
En cuanto al análisis SEM, la figura 5 muestra que los materiales sintetizados solidifican en pequeños nanocristales con forma esferoidal, con un tamaño promedio de $160 \mathrm{~nm}$, los que se asemejan al tamaño característico de los MOF. Los cristales obtenidos en DMF pura son más pequeños y dispersos que los obtenidos con el reemplazo del solvente por acetona, sin embargo la morfología continúa siendo esférica y con formación de agregados o clusters.

Para analizar la composición elemental de los nanocristales se realizó un mapeo de EDS para la muestra MOF100. Se observa, en la figura 6, que existe una distribución homogénea de $\mathrm{Zr}, 0$ y C en todo el material, como así también la presencia de átomos de $\mathrm{Cl}$ pertenecientes a la sal metálica.

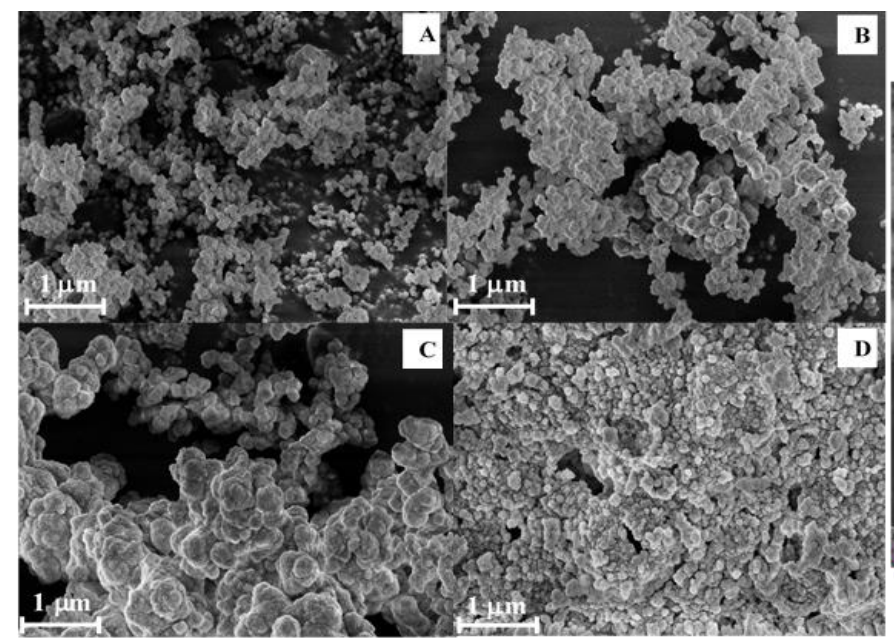

Figura 5 - Imágenes SEM de las muestras sintetizadas.

(A) $\mathrm{MOF}_{100 \%}$, (B) $\mathrm{MOF}_{75 \%}$, (C) $\mathrm{MOF}_{50 \%}$, (D) $\mathrm{MOF}_{25 \%}$.

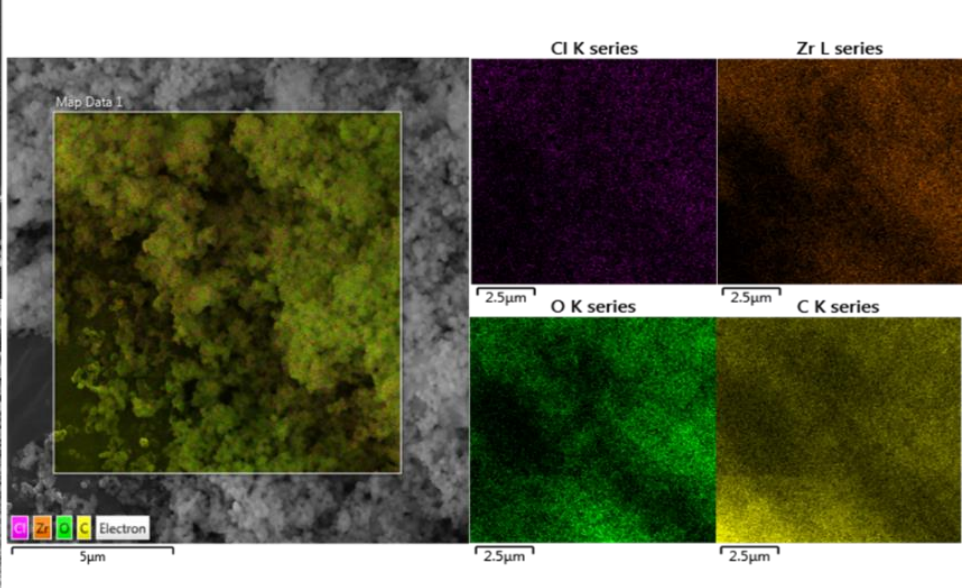

Figura 6 - Imágenes SEM-EDS de la muestra $\mathrm{MOF}_{100 \%}$.

La tabla 1 muestra el contenido de $\mathrm{Zr}(\% \mathrm{p} / \mathrm{p})$ que poseen los catalizadores sintetizados. Se puede observar que, en general, el porcentaje medido es próximo al teórico $(33 \% \mathrm{p} / \mathrm{p})$, con una pequeña disminución a medida que se fue reemplazando DMF.

Tabla 1: Análisis elemental, MP-AES

\begin{tabular}{|c|c|}
\hline Catalizador & Concentración [\% Zr p/p] \\
\hline $\mathrm{MOF}_{100 \%}$ & 28,38 \\
\hline $\mathrm{MOF}_{75 \%}$ & 25,85 \\
\hline $\mathrm{MOF}_{50 \%}$ & 23,38 \\
\hline $\mathrm{MOF}_{25 \%}$ & 24,88 \\
\hline
\end{tabular}

Las áreas superficiales de los materiales se muestran en la tabla 2. La mayor área corresponde al material con un $100 \%$ de DMF como solvente. A medida que se reemplazó el contenido de éste, existe una tendencia a la disminución del área superficial. Este efecto también puede ser observado con las imágenes de SEM donde las estructuras se vuelven más compactas y cerradas con el incremento de contenido de acetona. Al analizar el volumen de poro (tabla 2) se observa que el mismo disminuye a medida que se reemplaza la DMF; como el solvente es él que da la estructura interna del poro, al cambiarlo por acetona (molécula más pequeña) su tamaño se reduce. Mediante el análisis de las isotermas BET (Brunauer, Emmett y Teller) se determinó que los materiales se encuadran dentro de las isotermas de Tipo Ib, según la clasificación de la IUPAC, y que por el tamaño de poro, menor a $2 \mathrm{~nm}$, corresponde a los materiales microporosos (figura 7).
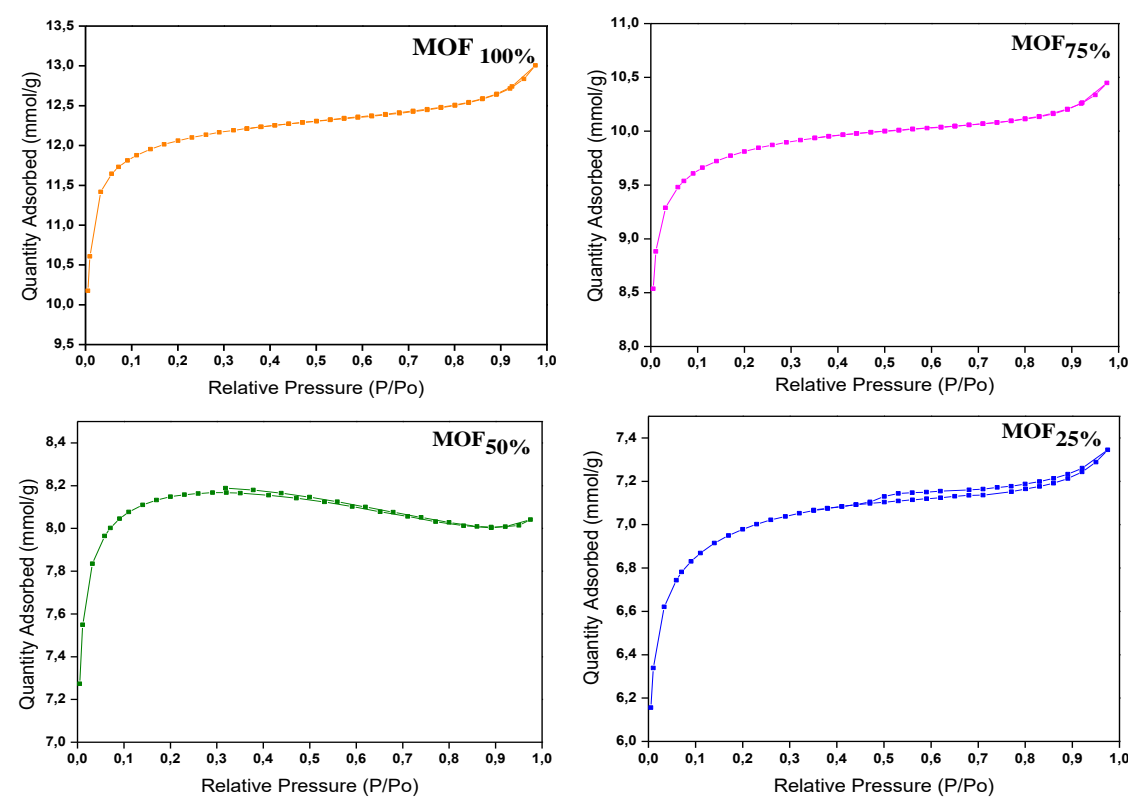

Tabla 2: Área-BET

\begin{tabular}{|l|c|c|c|}
\hline Catalizador & $\begin{array}{c}\text { Área BET } \\
\left(\mathrm{m}^{2} / \mathrm{g}\right)\end{array}$ & $\begin{array}{c}\text { Vol. de } \\
\text { poro } \\
\left(\mathrm{cm}^{3} / \mathrm{g}\right)\end{array}$ & $\begin{array}{c}\text { Tamaño } \\
\text { de poro } \\
(\mathrm{nm})\end{array}$ \\
\hline $\mathrm{MOF}_{100 \%}$ & 682,78 & 0,4508 & 1,2450 \\
\hline $\mathrm{MOF}_{75 \%}$ & 430,68 & 0,3622 & 1,2728 \\
\hline $\mathrm{MOF}_{50 \%}$ & 556,38 & 0,2787 & 1,1065 \\
\hline $\mathrm{MOF}_{25 \%}$ & 394,78 & 0,2546 & 1,2875 \\
\hline
\end{tabular}

Figura 7 - Isotermas adsorción - desorción 
Por último, en la figura 8 se exponen las gráficas de las deconvoluciones realizadas mediante el análisis de XPS. Con ello se confirma la presencia de los átomos de $\mathrm{Zr}$, C y O, que forman las estructura del MOF UiO-66. El Zr, se encuentra formando oxido metálico, con picos característicos del doblete, correspondiente a la energía de enlace del $\mathrm{Zr} 3 \mathrm{~d}$. El carbono se encuentra en la matriz orgánica (ácido tereftalico), con contribuciones de energía para C-C, C-O y $\mathrm{O}=\mathrm{C}-\mathrm{O}$; su pico satélite ubicado a altos valores de energía de enlace y el carbono ambiental que siempre está presente. Por último, el oxígeno a bajos valores de energía de enlace (530 eV) indica un entorno inorgánico, en este caso formando oxido con el Zr, mientras que a mayores energías de enlace se hace referencia a compuestos orgánico, formando parte de la estructura de ácido tereftalico.
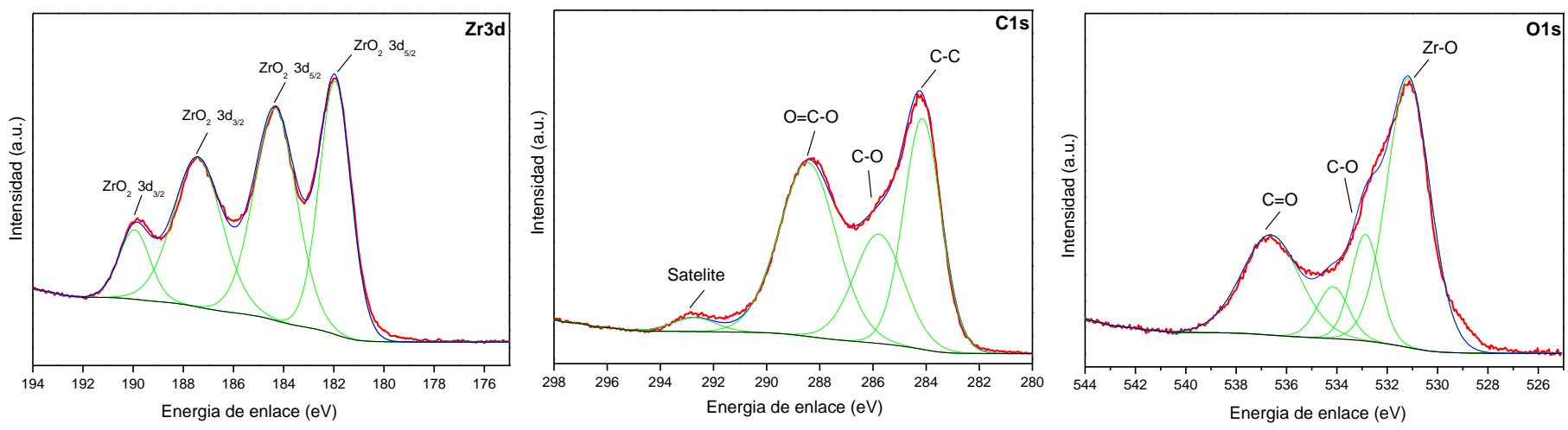

Figura 8 - Espectros XPS de la muestra $\mathrm{MOF}_{100 \%}$.

\section{Evaluación catalítica}

Los productos de reacción se determinaron por CG y posteriormente por espectroscopia de masa, para confirmar los compuestos obtenidos. Además del producto principal (levulinato de etilo), se comprobó la existencia de un producto secundario ( $\beta$-angélica lactona), proveniente de la deshidratación del ácido levulínico.

Con los datos obtenidos, una vez realizado el ajuste de las cromatografias, se pudo determinar los moles producidos del compuesto de interés, y calcular conversión, selectividad y rendimiento de la reacción de esterificación. Estos parametros son presentados en la figura 9 y tabla 3, donde se destaca que el $\mathrm{MOF}_{100 \%}$ es el que presenta la mayor converción y selectividad al producto deseado.

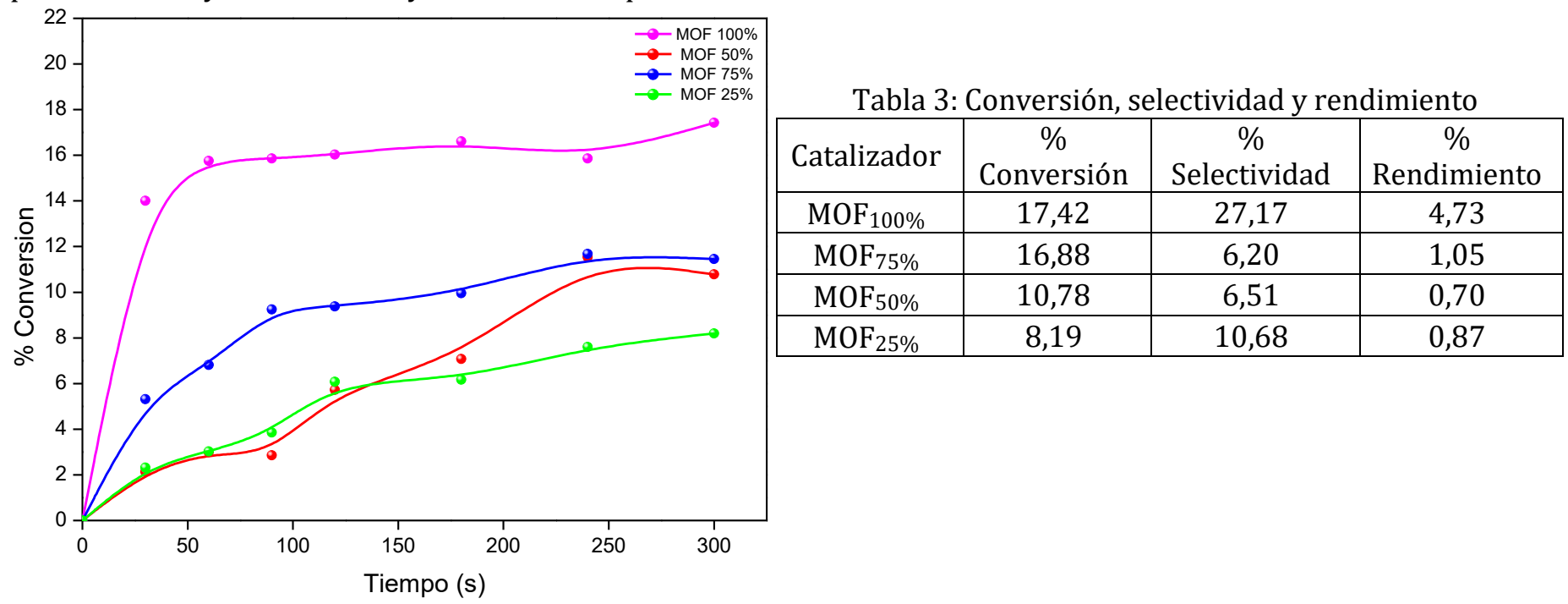

Figura 9 - Conversión de cada una de las reacción de la serie.

Finalmente, con los datos de conversión del reactivo, se pudo, mediante un ajuste, calcular las constantes de la velocidad de reacción para cada uno de los catalizadores utilizados. Debido a que uno de los reactivos (etanol) se encuentra en exceso con respecto al otro, la velocidad de reacción fue ajustada a una de pseudo primer orden, dependiente únicamente de la concentración del ácido levulínico (Ec. 1). En la tabla 4 se exponen los valores de cada constante (k) correspondiente a cada reacción catalítica.

$$
\frac{\mathrm{d}[\mathrm{A}]}{\mathrm{dt}}=\mathrm{k}[\mathrm{A}] \rightarrow \mathrm{k}^{*} \mathrm{t}=\mathrm{Ln} \frac{\left[\mathrm{A}_{0}\right]}{\left[\mathrm{A}_{\mathrm{t}}\right]}
$$

Ecuación 1: velocidad de reacción k: ctes de velocidad de reacción t: tiempo [A]: concentración de reactivo 
Tabla 4: constantes de velocidad de reacción

\begin{tabular}{|c|c|c|}
\hline Catalizador & $\mathrm{k}\left[\mathrm{min}^{-1}\right]$ & $\mathrm{R}^{2}$ \\
\hline $\mathrm{MOF}_{100 \%}$ & 0,000396303 & 0,5114 \\
\hline MOF $_{75 \%}$ & 0,000367428 & 0,9061 \\
\hline $\mathrm{MOF}_{50 \%}$ & 0,000325286 & 0,7585 \\
\hline $\mathrm{MOF}_{25 \%}$ & 0,000246733 & 0,9380 \\
\hline
\end{tabular}

\section{$>$ Conclusiones}

Se lograron obtener fases puras de MOF UiO-66 en condiciones solvotérmicas suaves, reemplazando hasta un $75 \% \mathrm{v} / \mathrm{v}$ de la tradicional dimetilformamida por acetona, con destacadas propiedades cristalinas y porosas. Si bien, el MOF obtenido mediante la síntesis con 100\% de DMF mostró las mejores propiedades cristalográficas, morfológicas y la mayor área superficial, todos los materiales podrían ser utilizados satisfactoriamente como soporte nanoestructurado capaz de alojar especies activas para diferentes aplicaciones.

En cuanto a la evaluación catalítica, el $\mathrm{MOF}_{100 \%}$ presentó la mayor actividad catalítica con una conversión del $17,42 \%$ y selectividad de $27,17 \%$. Esto se atribuye a que este material es él que presenta la mayor área superficial, por lo tanto posee más sitios activos disponibles para catalizar la reacción.

Finalmente, puede decirse que el remplazo de éstos catalizadores por los homogéneos en reacciones de esterificación, es una alternativa sumamente viable, que permite llevar adelante reacciones eco-compatibles con el medio, con una fácil separación de los productos de interés.

\section{Referencias}

[1] A. Corma, S. Iborra y A Velty. (2007). "Chemical Routes for the Transformation of Biomass into Chemicals". Chem. Rev. 107, 2411-2502. doi: 10.1021/cr050989d.

[2] Isao Ogino, Yukei Suzuki y Shin R.Mukai. (2018). Esterification of levulinic acid with ethanol catalyzed by sulfonated carbon catalysts: Promotional effects of additional functional groups. Catalysis Today. 314, 62-6962-69. doi: 10.1016/j.cattod.2017.10.001.

[3] Tianmeng Guo, Mo Qiu, Xinhua Qi. (2019). Selective conversion of biomass-derived levulinic acid to ethyl levulinate catalyzed by metal organic framework (MOF)-supported polyoxometalates. Catálisis Aplicada A: General 572 , 168-175. doi: 10.1016/j.apcata.2019.01.004.

[4] S. L. James, Chem. Soc. Rev. 32 (2003) 276-288.

[5] S. Kobayashi. (1999). Scandium Triflate in Organic Synthesis. Eur. J. Org. Chem. 15-27.

[6] J.H. Cavka, S. Jakobsen, U. Olsbye, N. Guillou, C. Lamberti, S. Bordiga and K.P. Lillerud. (2008). "A new zirconium inorganic building brick forming metal organic frameworks with exceptional stability"; Journal of the American Chemical Society 130. doi: 13850-13851.

[7] C. García Gil. (2016). Estudio de la actividad catalítica de Materiales híbridos metal-orgánico tipo uio-66 en reacciones de interés para la industria de la química fina. Escuela téncica superior ingenieros industriales Valencia.

[8] J. H. Cavka, S. Jakobsen, U. Olsbye, N. Guillou, C. Lamberti, S. Bordiga and K. P. Lillerud (2008). A New Zirconium Inorganic Building Brick Forming Metal Organic Frameworks with Exceptional Stability. J. Am. Chem. Soc. 130, 42. doi: 13850-13851.

[9] F.G.Cirujano, A.Corma, F.X.Llabrés i Xamena (2015). Conversion of levulinic acid into chemicals: Synthesis of biomass derived levulinate esters over Zr-containing MOFs. Chemical Engineering Science. 124, 52-60. doi: 10.1016/j.ces.2014.09.047.

[10] L. Lozano, C. Iglesias, B. Faroldi, M. Ulla y J. Zamaro. (2018). Efficient solvothermal synthesis of highly porous UiO-66 nanocrystals in dimethylformamide-free media. J Mater Sci 53, 1862-1873. doi:10.1007/s10853-017-1658-5.

[11] Y. Han, M. Liu, K. Li, Y. Zuo, Y. Wei, S. Xu, G. Zhang, C. Song, Z. Zhang and X. Guo. (2015). Facile synthesis of morphology and sizecontrolled zirconium metal-organic framework UiO-66: the role of hydrofluoric acid in crystallization. CrystEngComm 17, 6434-6440. Doi: 10.1039 / C5CE00729A.

[12] Z. Moghaddam, M. Kaykhaii, M. Khajeh, A. Oveisi. (2018). Synthesis of UiO-66-OH zirconium metal-organic framework and its application for selective extraction and trace determination of thorium in water samples by spectrophotometry. Molecular and Biomolecular Spectroscopy 194, 76-82. doi: 10.1016 / j.saa.2018.01.010.

[13] M. Katz, Z. Brown, Y. Colón, P. Siu, K. Scheidt, R. Snurr, J. Hupp and O. Farha. (2013). A facile synthesis of UiO-66, UiO-67 and their derivatives. Chem. Commun. 49, 9449. doi: 10.1039 / C3CC46105J. 